\title{
ON THE RELATIONSHIP BETWEEN DEBRIS DISKS AND PLANETS
}

\author{
Ágnes Kóspál ${ }^{1}$, David R. Ardila ${ }^{2}$, Attila MoóR ${ }^{3}$, ANd PÉter Ábrahám ${ }^{3}$ \\ ${ }^{1}$ Leiden Observatory, Leiden University, P.O. Box 9513, 2300 RA, Leiden, The Netherlands; kospal@ strw.leidenuniv.nl \\ 2 NASA Herschel Science Center, California Institute of Technology, Pasadena, CA 91125, USA \\ ${ }^{3}$ Konkoly Observatory of the Hungarian Academy of Sciences, P.O. Box 67, 1525 Budapest, Hungary \\ Received 2009 March 27; accepted 2009 June 19; published 2009 July 10
}

\begin{abstract}
Dust in debris disks is generated by collisions among planetesimals. The existence of these planetesimals is a consequence of the planet formation process, but the relationship between debris disks and planets has not been clearly established. Here we analyze Spitzer/MIPS 24 and $70 \mu \mathrm{m}$ data for 150 planet-bearing stars, and compare the incidence of debris disks around these stars with a sample of 118 stars around which planets have been searched for, but not found. Together they comprise the largest sample ever assembled to deal with this question. The use of survival analysis techniques allows us to account for the large number of nondetections at $70 \mu \mathrm{m}$. We discovered 10 new debris disks around stars with planets and one around a star without known planets. We found that the incidence of debris disks is marginally higher among stars with planets, than among those without, and that the brightness of the average debris disk is not significantly different in the two samples. We conclude that the presence of a planet that has been detected via current radial velocity techniques is not a good predictor of the presence of a debris disk detected at infrared wavelengths.
\end{abstract}

Key words: circumstellar matter - infrared: stars - Kuiper Belt - planetary systems

Online-only material: machine-readable table

\section{INTRODUCTION}

As of the end of 2008, 228 extrasolar planets around 193 stars had been discovered using radial velocity techniques. Most of them are believed to be Jupiter-like gas giants, although some may be lower-mass rocky planets (Udry \& Santos 2007). According to the core accretion model, planets are formed by the agglomeration of rocky planetesimals into solid cores (Lissauer 2005). For gas giants, the core accretes a gaseous envelope. The planets may migrate toward the star due to interactions with the disk material (Lin et al. 2000), and strong planetplanet gravitational scattering may significantly modify orbital parameters (Raymond et al. 2008).

It is also known that many main-sequence stars display infrared and/or submillimeter excess emission due to the presence of debris dust grains (e.g., Trilling et al. 2008). These grains are replenished by collisions among planetesimals, leftovers of the planet formation process (Backman \& Paresce 1993). Planets orbiting the star can stir the planetesimal population and initiate a collisional cascade, resulting in dust production. The morphology of the disk might be gravitationally shaped by planets orbiting interior to the disk (Chiang et al. 2009). The common origin of planets and debris disks suggests that a connection may exist between the presence of planets and planetesimals.

The relationship between debris disks and planets can be studied by comparing the incidence of excess emission due to debris dust between samples of stars with and without planets. An overview of such studies is presented in Table 1. For Sunlike stars, e.g., Beichman et al. $(2005,2006)$ hinted at a positive correlation between debris disks and planets; Moro-Martín et al. (2007) found no significant correlation; and Trilling et al. (2008) claimed that although excess rates for the planet sample are higher, both samples' excess rates are consistent at the $1 \sigma$ level. More conclusive results can only be based on larger samples than used in those studies.

Here we use data from the Spitzer Space Telescope archive at 24 and $70 \mu \mathrm{m}$ to compare the incidence of debris dust around stars with planets (SWPs) discovered/confirmed using radial velocity techniques and those where radial velocity measurements did not indicate planetary companions (stars without planets, SWOPs). We use all public data constructing the largest sample ever considered in attacking this question.

\section{OBSERVATIONS \\ 2.1. Sample Selection}

We considered 193 SWPs discovered/confirmed using radial velocity measurements as of 2008 December. ${ }^{4}$ Out of these, 150 have publicly available Spitzer/MIPS 24 and/or $70 \mu \mathrm{m}$ measurements (all of them were observed at $70 \mu \mathrm{m}, 83$ of them at $24 \mu \mathrm{m})$. These constitute our planet-bearing sample. For SWOPs, we used those stars that were present in the Keck, Lick, and Anglo-Australian Telescope planet search programs (Wright et al. 2004; Valenti \& Fischer 2005), but around which no planets were found. These stars have no planets with radial velocity semi-amplitudes $K>30 \mathrm{~m} \mathrm{~s}^{-1}$ and orbital periods shorter than four years, and the radial velocity measurements have a typical Doppler precision of $3 \mathrm{~m} \mathrm{~s}^{-1}$. We based our SWOPs sample on two Spitzer surveys of nearby stars (Pid 41 and Pid 2324) and discarded some stars where close-by objects would have contaminated the MIPS photometry. The resulting SWOPs sample includes 118 objects (all of them were observed at $24 \mu \mathrm{m}$, all but one at $70 \mu \mathrm{m}$, see Table 2). Both samples contain stars with spectral types between F3 and M3. While the majority are main-sequence stars, a few subgiants are also present, and the SWPs sample also contains four giants. As age is a key parameter in debris disk evolution (Wyatt 2008), we checked whether the ages differed significantly in the two samples. We calculated two-sample tests (Gehan's generalized Wilcoxon test, logrank test, Peto \& Peto generalized Wilcoxon test, and Peto \& Prentice generalized Wilcoxon test), and found

\footnotetext{
4 Regularly updated lists of extrasolar planets can be found at http://www.exoplanets.org and at http://www.exoplanet.eu.
} 
Table 1

Spitzer-Based Surveys of Debris Disks Around Main-Sequence Stars (Stars with Excess/Total Observed Sample)

\begin{tabular}{|c|c|c|c|c|c|}
\hline \multirow[t]{2}{*}{ Reference } & \multirow[t]{2}{*}{ Sample } & \multicolumn{2}{|c|}{$24 \mu \mathrm{m}$} & \multicolumn{2}{|c|}{$70 \mu \mathrm{m}$} \\
\hline & & SWP & SWOP & SWP & SWOP \\
\hline Beichman et al. (2005) & FGK & $0 / 25(0 \%)$ & $\ldots$ & $6 / 25(24 \%)$ & $\ldots$ \\
\hline Bryden et al. (2006) & FGK & $1 / 12(8 \%)$ & $0 / 57(0 \%)$ & $1 / 12(8 \%)$ & $6 / 57(10 \%)$ \\
\hline Beichman et al. (2006) & FGKM & $\ldots$ & $5 / 88(6 \%)$ & $\ldots$ & $12 / 88(14 \%)$ \\
\hline Su et al. (2006) & A & $\ldots$ & $50 / 155(32 \%)$ & $\ldots$ & $44 / 134(33 \%)$ \\
\hline Gautier et al. (2007) & M & $0 / 3(0 \%)$ & $0 / 59(0 \%)$ & $0 / 3(0 \%)$ & $0 / 38(0 \%)$ \\
\hline Moro-Martín et al. (2007) & FGK & $0 / 9(0 \%)$ & $2 / 99(2 \%)$ & $1 / 9(11 \%)$ & $9 / 99(9 \%)$ \\
\hline Trilling et al. (2008) & FGK & $3 / 45(7 \%)$ & $4 / 139(3 \%)$ & $10 / 48(21 \%)$ & $22 / 148(15 \%)$ \\
\hline Carpenter et al. (2009) & FGK & $0 / 10(0 \%)$ & $41 / 299(14 \%)$ & $2 / 10(20 \%)$ & $19 / 299(6 \%)$ \\
\hline present work & FGKM & $3 / 83(4 \%)$ & $1 / 118(1 \%)$ & $22 / 150(15 \%)$ & $17 / 117(15 \%)$ \\
\hline
\end{tabular}

Table 2

Photospheric Predictions and MIPS Photometry

\begin{tabular}{|c|c|c|c|c|c|c|c|c|c|c|c|c|c|c|c|c|}
\hline Name & Type & $\begin{array}{l}\text { Age } \\
(\mathrm{Gyr})\end{array}$ & $\begin{array}{c}\text { Age } \\
\text { ref }\end{array}$ & {$[\mathrm{Fe} / \mathrm{H}]$} & $\begin{array}{l}F_{24}^{\text {pred }} \\
(\mathrm{mJy})\end{array}$ & $\begin{array}{c}F_{24}^{\mathrm{obs}} \\
(\mathrm{mJy})\end{array}$ & $\begin{array}{c}\sigma_{24} \\
(\mathrm{mJy})\end{array}$ & $R_{24}$ & $\chi_{24}$ & $\mathrm{IRE}_{24}{ }^{\mathrm{a}}$ & $\begin{array}{l}F_{70}^{\text {pred }} \\
(\mathrm{mJy})\end{array}$ & $\begin{array}{c}F_{70}^{\mathrm{obs}} \\
(\mathrm{mJy})\end{array}$ & $\begin{array}{c}\sigma_{70} \\
(\mathrm{mJy})\end{array}$ & $R_{70}$ & $\chi_{70}$ & $\operatorname{IRE}_{70} \mathrm{~b}$ \\
\hline HD 142 & SWP & 3.120 & 1 & 0.0875 & 109.5 & 119.6 & 4.90 & 1.09 & 2.06 & $\mathrm{NO}$ & 11.92 & 18.16 & 2.73 & 1.52 & 2.28 & $\mathrm{NO}$ \\
\hline HD 166 & SWOP & 0.200 & 2 & -0.0600 & 134.6 & 157.4 & 6.45 & 1.17 & 3.53 & YES & 14.68 & 102.9 & 8.42 & 7.01 & 10.48 & YES \\
\hline HD 1237 & SWP & 0.625 & 3 & 0.1100 & 79.40 & 82.61 & 3.39 & 1.04 & 0.95 & NO & 8.67 & 11.07 & 1.68 & 1.28 & 1.43 & NO \\
\hline HD 1581 & SWOP & 4.840 & 1 & -0.2025 & 567.6 & 527.1 & 21.61 & 0.93 & -1.87 & $\mathrm{NO}$ & 62.13 & 74.40 & 7.93 & 1.20 & 1.55 & NO \\
\hline HD 3651 & SWP & 8.200 & 4 & -0.0540 & 187.8 & 188.2 & 7.72 & 1.00 & 0.05 & $\mathrm{NO}$ & 20.51 & 15.49 & 5.12 & 0.76 & -0.98 & NO \\
\hline HD 3823 & SWOP & 7.200 & 1 & -0.3100 & 110.8 & 111.8 & 4.58 & 0.01 & 0.20 & $\mathrm{NO}$ & 12.14 & $<13.49$ & $\ldots$ & $<1.11$ & $\ldots$ & UPL \\
\hline HD 3795 & SWOP & 11.600 & 1 & -0.6425 & 130.0 & 135.7 & 5.56 & 1.04 & 1.03 & $\mathrm{NO}$ & 14.30 & 13.92 & 2.49 & 0.97 & -0.15 & NO \\
\hline HD 4208 & SWP & 7.760 & 1 & -0.2600 & 24.81 & $\ldots$ & $\ldots$ & $\ldots$ & $\ldots$ & $\ldots$ & 2.72 & $<5.35$ & $\ldots$ & $<1.96$ & $\ldots$ & UPL \\
\hline HD 4308 & SWP & 8.680 & 1 & -0.3933 & 73.67 & 74.73 & 3.06 & 1.01 & 0.35 & $\mathrm{NO}$ & 8.08 & $<13.00$ & $\ldots$ & $<1.61$ & $\ldots$ & UPL \\
\hline
\end{tabular}

Notes.

${ }^{a}$ Infrared excess at $24 \mu \mathrm{m}$. SWPs with excess: HD 10647, HD 22049, HD 69830. SWOPs with excess: HD 166.

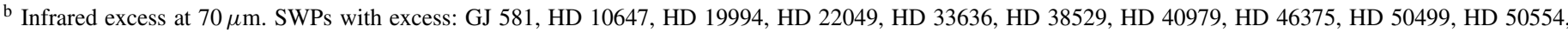

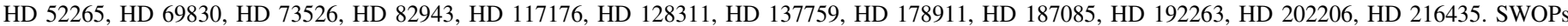

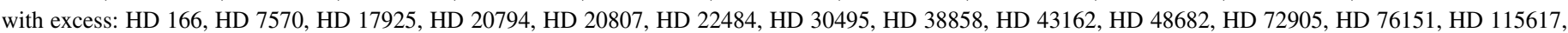
HD 118972, HD 158633, HD 206860, HD 207129.

(This table is available in its entirety in a machine-readable form in the online journal. A portion is shown here for guidance regarding its form and content.)

that the age distributions do not differ (mean ages are $5.317 \mathrm{Gyr}$ for SWPs and 4.648 Gyr for SWOPs). The same holds for the effective temperatures (mean effective temperatures are $5460 \mathrm{~K}$ for SWPs and $5550 \mathrm{~K}$ for SWOPs).

\subsection{Data Processing}

\subsubsection{Data Reduction}

From the Spitzer archive we downloaded BCD files reduced with the SSC pipeline version 16.0 or 16.1 . At $70 \mu \mathrm{m}$, we used GeRT (version S14.0 v1.1 [060415]) to do column mean subtraction and time filtering on the BCD files. We used Mopex (version 18.1.5) to create mosaics for 24 and $70 \mu \mathrm{m}$ images and obtained aperture photometry using IDL. At $24 \mu \mathrm{m}$, we used an aperture radius of $7^{\prime \prime}$, sky annulus between $40^{\prime \prime}$ and $50^{\prime \prime}$, and aperture correction of 1.61 ; at $70 \mu \mathrm{m}$, we used an aperture radius of $8^{\prime \prime}$, sky annulus between $39^{\prime \prime}$ and $65^{\prime \prime}$, and aperture correction of 3.70, appropriate for a 10,000 K blackbody. After identifying stars with $70 \mu \mathrm{m}$ excess, we recalculated their fluxes with an aperture correction of 3.83, appropriate for a $60 \mathrm{~K}$ blackbody. Appropriate color corrections were also performed. Thanks to the small aperture, nearby sources had no effect on our photometry. At $24 \mu \mathrm{m}$, the aperture was placed around the centroid of each star, while at $70 \mu \mathrm{m}$, the aperture was placed at the same position as for the $24 \mu \mathrm{m}$ image. If a $24 \mu \mathrm{m}$ position was not available, we used fixed coordinates. For sources brighter than $100 \mathrm{mJy}$ and having signal-to-noise ratio of $\geqslant 11$, we checked their spatial extent by comparing the images to a stellar point-spread function (PSF). For extended objects (HD 10647, HD 38858, HD 207129 , HD 48682, and HD 115617) photometry was extracted by fitting a PSF broadened by an appropriate two-dimensional Gaussian. The fluxes of $\epsilon$ Eri were taken from Backman et al. (2009).

\subsubsection{Photosphere Prediction}

We collected near-infrared photometry from the 2MASS AllSky Catalog of Point Sources (Skrutskie et al. 2006), Morel \& Magnenat (1978), and Ducati (2002), $8.28 \mu \mathrm{m}$ fluxes from the MSX Infrared Point-Source Catalog (Egan et al. 2003), and $12 \mu \mathrm{m}$ fluxes from the IRAS catalog of point sources (with appropriate color correction). Spectral types, effective temperatures, and metallicities came from the NASA Star and Exoplanet Database. ${ }^{5}$ For each FGK star we selected the model from the ATLAS9 grid of model atmospheres (Castelli \& Kurucz 2003) that has the closest metallicity, $\log g=4.5$, and interpolated between the two closest models in effective temperature. This model spectrum was then scaled to the nearand mid-infrared photometric points, and its value at $23.68 \mu \mathrm{m}$ and at $71.42 \mu \mathrm{m}$ was calculated. M stars were similarly fitted with NextGen model atmospheres (Hauschildt et al. 1999). The resulting photospheric fluxes $\left(F_{24}^{\text {pred }}, F_{70}^{\text {pred }}\right)$ are presented in Table 2.

\footnotetext{
5 http://nsted.ipac.caltech.edu/
} 

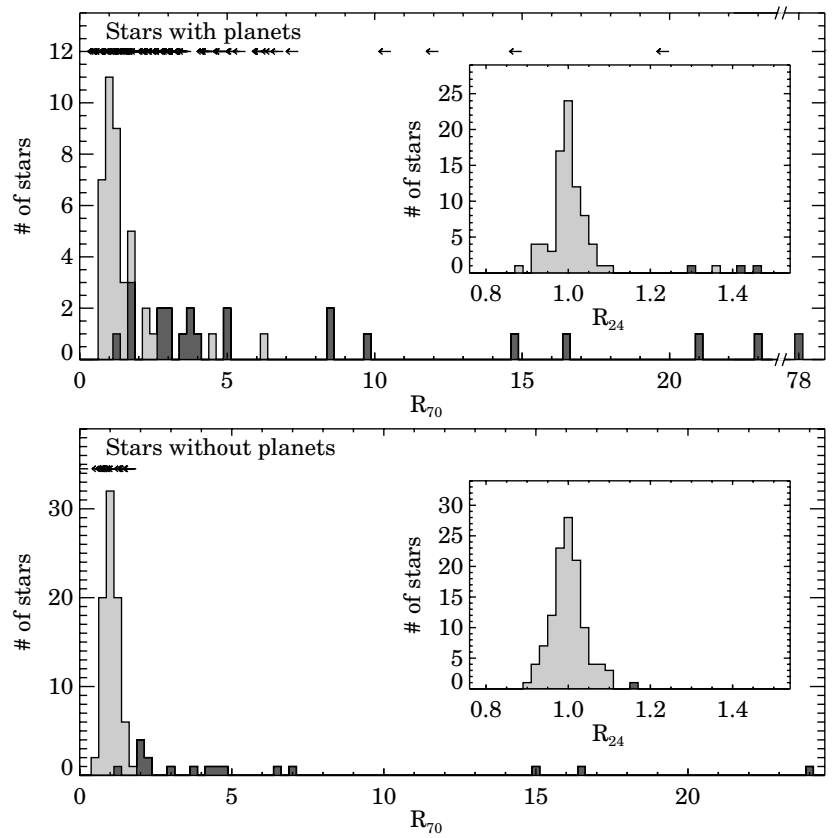

Figure 1. Distribution of observed-to-predicted flux ratios at 70 and $24 \mu \mathrm{m}\left(\mathrm{R}_{70}\right.$ in the big graphs, and $R_{24}$ in the small insets). Stars with significant $(>3 \sigma)$ excess are indicated with dark shades. Upper limits are indicated with left arrows.

\subsubsection{Empirical Correction}

To test our photometric predictions, we considered the ratio of the observed and predicted flux $\left(R_{24}, R_{70}\right)$ as a function of the logarithm of the observed brightness $\left(\log \left(F_{24}^{\mathrm{obs}}\right)\right.$, $\left.\log \left(F_{70}^{\mathrm{obs}}\right)\right)$. We found that $F_{24}^{\mathrm{obs}}$ tends to be systematically higher than $F_{24}^{\text {pred }}$ for bright stars. At $70 \mu \mathrm{m}$ no such trend could be seen. At $24 \mu \mathrm{m}$ the points could be well fitted with a linear relationship, which we used to correct the observed fluxes (note that Gordon et al. 2007 found a similar dependence of the $70 \mu \mathrm{m}$ aperture photometry on source brightness, see their Figures 3 and 4 ). The correction was always less than $6 \%$. We found no dependence of the corrected flux on the effective temperature. This is in agreement with Engelbracht et al. (2007), though is in contrast with Beichman et al. (2006). Our Spitzer/MIPS 24 and $70 \mu \mathrm{m}$ photometry, together with individual uncertainties (including those of the absolute flux calibration of the MIPS instrument, $4 \%$ at 24 and $7 \%$ at $70 \mu \mathrm{m}$; see the MIPS Data Handbook) can be seen in Table 2 .

\section{RESULTS}

\subsection{Identification of Debris Disks}

In all $24 \mu \mathrm{m}$ observations the target was detected at $>3 \sigma$ level, where $\sigma$ is the flux uncertainty. At $70 \mu \mathrm{m}, 59$ out of 150 SWPs, and 97 out of 117 SWOPs were detected at $>3 \sigma$ level. For the nondetections at $70 \mu \mathrm{m}$, upper limits were calculated as $3 \sigma_{70}$ if the observed flux in the aperture $\left(F_{70}^{\text {obs }}\right)$ was negative, and $F_{70}^{\mathrm{obs}}+3 \sigma_{70}$ if positive (Carpenter et al. 2009). A star has significant excess if $\chi_{70}=\left(F_{70}^{\text {obs }}-F_{70}^{\text {pred }}\right) / \sigma_{70}>3$ (Table 2$)$. In the SWPs sample, three stars have significant excess at $24 \mu \mathrm{m}$, and 22 stars at $70 \mu \mathrm{m}$. In the SWOPs sample, one star shows significant $24 \mu \mathrm{m}$ excess, while 17 stars have significant excess at $70 \mu \mathrm{m}$. The names of stars with excess are listed in the notes to Table 2.

Figure 1 shows the histogram of observed-to-photospheric flux ratios at 24 and $70 \mu \mathrm{m}\left(R_{24}, R_{70}\right)$. At both wavelengths
Table 3

Newly Identified Debris Disks

\begin{tabular}{lrrrrrr}
\hline \hline \multicolumn{1}{c}{ Name } & $\begin{array}{c}F_{70}^{\text {pred }} \\
(\mathrm{mJy})\end{array}$ & \multicolumn{1}{c}{$\begin{array}{c}F_{70}^{\text {obs }} \\
(\mathrm{mJy})\end{array}$} & $\begin{array}{c}\sigma_{70} \\
(\mathrm{mJy})\end{array}$ & \multicolumn{1}{c}{$R_{70}$} & $\chi_{70}$ & $L_{\text {dust }} / L_{*}$ \\
\hline GJ 581 $^{\mathrm{a}}$ & 5.98 & 16.47 & 2.94 & 2.753 & 3.567 & $1.2 \times 10^{-5}$ \\
HD 40979 $^{\mathrm{a}}$ & 5.11 & 14.24 & 2.73 & 2.784 & 3.346 & $1.2 \times 10^{-5}$ \\
HD 43162 $^{\mathrm{b}}$ & 10.01 & 19.82 & 2.95 & 1.980 & 3.329 & $1.0 \times 10^{-5}$ \\
HD 46375 $^{\mathrm{a}}$ & 3.23 & 74.68 & 19.04 & 23.102 & 3.753 & $2.3 \times 10^{-4}$ \\
HD 50499 $^{\mathrm{a}}$ & 3.62 & 10.70 & 2.16 & 2.957 & 3.273 & $1.3 \times 10^{-5}$ \\
HD 73526 $^{\mathrm{a}}$ & 0.91 & 19.11 & 5.38 & 20.943 & 3.382 & $1.7 \times 10^{-4}$ \\
HD 137759 & 455.52 & 615.53 & 44.08 & 1.351 & 3.630 & $4.3 \times 10^{-6}$ \\
HD 178911 & 2.23 & 11.43 & 1.88 & 5.119 & 4.891 & $3.6 \times 10^{-5}$ \\
HD 187085 & 3.45 & 13.34 & 2.81 & 3.867 & 3.520 & $1.8 \times 10^{-5}$ \\
HD 202206 & 2.00 & 29.51 & 3.77 & 14.735 & 7.290 & $1.1 \times 10^{-4}$ \\
HD 216435 & 10.88 & 41.74 & 4.04 & 3.836 & 7.636 & $2.0 \times 10^{-5}$ \\
\hline
\end{tabular}

Notes.

a Stars with planets.

b Stars without planets.

the distribution peaks at $R \approx 1$, and can be approximated with a Gaussian of $\sigma=4 \%$ at $24 \mu \mathrm{m}$ (the error budget is dominated by the uncertainty of the absolute flux calibration) and $\sigma=24 \%$ at $70 \mu \mathrm{m}$ (the photometry is confusion noise limited at this wavelength). Out of the 39 stars with excess, shown with dark shades in Figure 1, 28 were known in the literature to harbor debris disks. For the remaining 11 our work shows the first indication for a debris disk (Table 3).

\subsection{Survival Analysis}

At $24 \mu \mathrm{m}$, all observed stars were detected at several $\sigma$ level. Thus, the distribution of excesses can be compared with traditional two-sample tests, which show that SWPs and SWOPs do not differ. This is due to the fact that the overwhelming majority of stars exhibit pure photospheric emission at $24 \mu \mathrm{m}$.

The $70 \mu \mathrm{m}$ data set is censored - there are upper limits-and survival analysis is required to compare the two distributions (e.g., Moro-Martín et al. 2007). The Kaplan-Meier (KM) estimator gives the cumulative distribution of a statistical variable taking into account the upper limits (e.g., Feigelson $\&$ Nelson 1985). To use survival analysis, the censoring should not depend on the variable itself. This condition can be fulfilled by using the observed-to-photospheric flux ratio $\left(R_{24}\right.$ and $\left.R_{70}\right)$ as the variable. In average SWPs are farther than SWOPs. As a consequence, the former tend to be apparently fainter than the latter, resulting in more nondetections in the SWPs sample, introducing Malmquist bias. This bias is eliminated by using the distance-independent observed-to-photospheric flux ratio.

Figure 2 presents the KM estimators for our SWPs and SWOPs samples. Calculations were done with the ASURV Rev. 1.2 package (Lavalley et al. 1992), which implements the methods presented in Feigelson \& Nelson (1985). As with the two-sample tests, the KM estimators show that at $24 \mu \mathrm{m}$ there is no difference between the distribution of excesses of SWPs and of SWOPs. At $70 \mu \mathrm{m}$, however, the two KM curves are marginally different. The largest distance between the curves occurs at $R_{70}=1.5$. The values at this point are $0.273 \pm$ 0.045 and $0.155 \pm 0.034$ for SWPs and SWOPs, respectively, a $2.1 \sigma$ difference. Thus, SWPs tend to have excesses slightly more often than SWOPs. Other statistical tests also suggest that the distributions are marginally different. For example, Gehan's generalized Wilcoxon test indicates that the probability of the null hypothesis-that SWPs and SWOPs have the same incidence of debris disks-is $13 \%$. Other tests give probabilities 

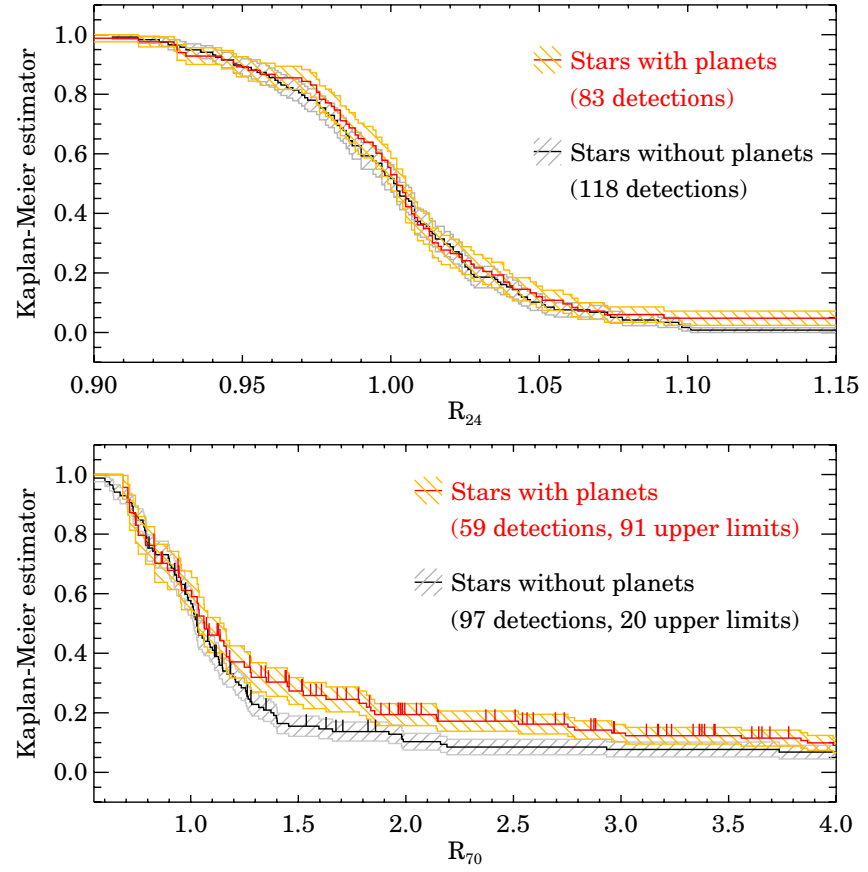

Figure 2. KM estimators for our two samples as a function of observed-tophotospheric flux ratio at $24 \mu \mathrm{m}$ (upper panel) and at $70 \mu \mathrm{m}$ (lower panel). Upper limits are indicated by vertical dashes. The KM estimator at a certain flux ratio gives the fraction of stars having a flux ratio larger than that value.

between $20 \%$ and $40 \%$. To increase the significance of the obtained $2.1 \sigma$ difference to $5 \sigma$ would require about five times more objects in each sample. The mean excess at $70 \mu \mathrm{m}$, as given by the area under the KM estimator curves is $2.975 \pm$ 0.732 for SWPs, and $1.725 \pm 0.273$ for SWOPs. Though disks around SWPs tend to be brighter, the difference is only $1.6 \sigma$. The results do not change significantly if we discard the four giant stars from the SWPs sample.

The common way in the literature to calculate detection rates is to divide the number of stars with excess by the number of all observed stars (Table 1). At $70 \mu \mathrm{m}$ this method gives a detection rate of $15 \%$ for both SWPs and SWOPs. However, if we were to divide by the number of detected sources only, we would conclude that $37 \%$ of the SWPs have excesses, compared to $18 \%$ of the SWOP sample. The discrepancy of these methods is related to the presence of upper limits, which are correctly handled in our survival analysis.

\section{DISCUSSION}

It is an open question whether the orbital parameters of the planets correlate with the presence of debris disks (Chiang et al. 2009). We compared stars with and without excesses within the SWP sample. The two-sample tests mentioned above show that the planets' semimajor axes follow the same distribution in the two groups, while eccentricities and masses are slightly higher for stars having both planets and debris disks than those without debris disks, although the differences are not statistically significant.

The mean metallicities for our stars are $[\mathrm{Fe} / \mathrm{H}]=0.103 \pm$ 0.018 and $-0.097 \pm 0.024$ for SWPs and SWOPs, respectively. As expected (Santos et al. 2001; Valenti \& Fischer 2005), twosample tests show the metallicity distributions to be significantly different in our samples. To check whether there are differences in the distribution of excesses between metal-rich and metal- poor stars, we analyzed the SWPs and SWOPs samples separately. For each sample, we compared $R_{70}$ between the bottom and top thirds of the metallicity distributions, using KM estimators. We found that metallicity does not correlate with the infrared excess in either sample, confirming results obtained for smaller samples (Greaves et al. 2006; Beichman et al. 2005; Bryden et al. 2006). While metallicity is one of the strongest predictors for the presence of giant planets (Valenti \& Fischer 2005), it does not predict the presence of debris disks.

Moro-Martín et al. (2007) argued that the larger metallicity of SWPs implies that more planetesimals were formed early in those systems but that most were expelled due to the orbital evolution of the giant planets. The planetesimal configuration is then the same that would occur in an average metallicity SWOPs. This implies that debris disks are more common around metalrich stars without planets, which is in contrast to the observations presented here.

Around a $1 L_{\odot}$ solar-type star, a narrow ring of dust particles radiating as blackbodies would have the peak of its emission $\left(F_{v}\right)$ at $24 \mu \mathrm{m}$ if located at $1.7 \mathrm{AU}$ from the star. The gravitational influence of giant planets extends at most to the 3:1 mean motion resonance (e.g., Moro-Martín \& Malhotra 2005). For our sample, where the average semimajor axis is $1.4 \mathrm{AU}$, this would be $2.9 \mathrm{AU}$. Thus, these planets might stir the planetesimals located within a belt similar to the asteroid belt in our solar system. However, our results show that $24 \mu \mathrm{m}$ excess is rare in both SWPs and SWOPs. Moreover, not all stars exhibiting $24 \mu \mathrm{m}$ excess have dust within this region: the warmest dust present in these systems is $60 \mathrm{~K}$ dust at a radius of $25 \mathrm{AU}$ for HD 10647 (Liseau et al. 2008), $120 \mathrm{~K}$ dust at 3 AU for HD 22049 (Backman et al. 2009), $245 \mathrm{~K}$ dust at 1 AU for HD 69830 (Lisse et al. 2007), and $87 \mathrm{~K}$ dust at $9.1 \mathrm{AU}$ for HD 166 (Trilling et al. 2008). This implies that warm debris dust is intrinsically rare in both SWPs and SWOPs, thus the physical reason of this rarity is probably unrelated to the planets. With so few stars with $24 \mu \mathrm{m}$ excess, the KM estimators cannot rule out any difference between the SWPs and the SWOPs samples. Our observations do not preclude that there are differences between the two samples in the distribution of asteroid belt-type debris disks fainter than our detection limit at $24 \mu \mathrm{m}\left(L_{\text {dust }} / L_{*} \approx 10^{-5}\right.$, as calculated from Equation (11) of Wyatt 2008).

Debris dust peaking at $70 \mu \mathrm{m}$ is usually too far from the planets considered here to be affected by their presence. Dust distances for debris disk systems with excesses at $70 \mu \mathrm{m}$ are mostly larger than 4-5 AU (Trilling et al. 2008). The null hypothesis is consistent with that measurement. Trilling et al. (2008) argued that stars with planets are typically farther and in more confused regions of the sky, and suggest that dust around them may be more common than what detections indicate. In our sample, the median distance to the SWPs is $35 \mathrm{pc}$, compared to the $15 \mathrm{pc}$ for the SWOPs, and two-sample tests reveal these distances to be significantly different. However, our main analysis quantity is the distance-independent $R$, and we explicitly include information about sky confusion noise in the form of upper limits, which are taken into account in the survival analysis. Therefore, our results do not support the presence of a large population of undiscovered debris disks around SWPs.

At $70 \mu \mathrm{m}$, our detection limit (Equation (11) of Wyatt 2008) is $L_{\text {dust }} / L_{*} \approx 10^{-6}$. For these bright debris disks, the null hypothesis implies that the population of planets at distances where the $70 \mu \mathrm{m}$ emission comes from is the same for SWPs and the SWOPs. The marginally higher incidence of debris disks around SWPs suggests that in these systems an outer planet 
might also exist, which stirs up the planetesimals producing a cold debris disk.

The model by Wyatt et al. (2007) for A-type stars suggests that the $70 \mu \mathrm{m}$ emission should be larger for SWPs than for SWOPs, due to the larger mass of the protoplanetary disk in the former. They speculate that a similar trend holds for Sunlike stars. We found that the brightest debris disks in our SWPs sample are 2-3 times brighter than those in the SWOPs sample, but the mean brightnesses are only slightly higher in the SWPs sample at a $1.6 \sigma$ level. Our results do not contradict with the prediction of Wyatt et al. (2007), although the difference we found is less pronounced than that expected for A-type stars. Note that disk mass is not the only variable that controls the likelihood of planet formation: disk lifetime, metallicity, and surface density distribution are also important.

\section{SUMMARY AND CONCLUSIONS}

Prior to this study, only a dozen stars were known to harbor both planets and debris disks. Our 10 new debris disks found around planet-bearing stars at $70 \mu \mathrm{m}$ doubled this number. We analyze a sample of planet-host stars at least three times larger than any previous studies and a carefully chosen control sample of stars without planets that is comparable in size. We have found that the incidence of debris disks (measured at $70 \mu \mathrm{m}$ ) is only marginally higher among stars with planets, than among those without. This result suggests the possibility thatif debris production is primarily due to stirring by planetsthe planet population at large radii (larger than what current radial velocity surveys can find) is comparable in both samples (although there are other possibilities for debris production, such as planetesimal self-stirring, or external perturbations). We found that the brightness of the average debris disk is not significantly different in the two samples. We could not discover any clear correlation between the planets' orbital parameters or the parent stars' metallicity and the presence of debris dust.

This work is based on observations made with the Spitzer Space Telescope, which is operated by the Jet Propulsion Laboratory, California Institute of Technology under a contract with NASA. Á.K. acknowledges support from the Spitzer Visiting Graduate Student Fellowship and from the Netherlands Organization for Scientific Research (NWO). The authors thank
L. Balázs for useful discussions about statistics and the referee for his/her comments. This work was partly supported by the Hungarian Research Fund OTKA K62304.

Facilities: Spitzer

\section{REFERENCES}

Backman, D., \& Paresce, F. 1993, Protostars and Planets III, ed. E. H. Levy \& J. I. Lunine (Tucson, AZ: Univ. Arizona Press), 1253

Backman, D., et al. 2009, ApJ, 690, 1522

Beichman, C. A., et al. 2005, ApJ, 622, 1160

Beichman, C. A., et al. 2006, ApJ, 652, 1674

Bryden, G., et al. 2006, ApJ, 636, 1098

Carpenter, J. M., et al. 2009, ApJS, 181, 197

Castelli, F., \& Kurucz, R. L. 2003, in Proc. IAU Symp. 210, Modelling of Stellar Atmospheres, ed. N. Piskunov, W. W. Weiss, \& D. F. Gray (Dordrecht: Kluwer), 20

Chiang, E., Kite, E., Kalas, P., Graham, J. R., \& Clampin, M. 2009, ApJ, 693, 734

Ducati, J. R. 2002, VizieR Online Data Catalog, 2237 (http://cdsads.u strasbg.fr/ abs/2002yCat.2237....OD)

Egan, M. P., et al. 2003, Air Force Research Laboratory Technical Report AFRL-VS-TR-2003-1589

Engelbracht, C. W., et al. 2007, PASP, 119, 994

Feigelson, E. D., \& Nelson, P. I. 1985, ApJ, 293, 192

Gautier, Th. N., et al. 2007, ApJ, 667, 527

Gordon, K. D., et al. 2007, PASP, 119, 1019

Greaves, J. S., Fischer, D. A., \& Wyatt, M. C. 2006, MNRAS, 366, 283

Hauschildt, P. H., Allard, F., \& Baron, E. 1999, ApJ, 512, 377

Lavalley, M., Isobe, T., \& Feigelson, E. 1992, in ASP Conf. Ser. 25, Astronomical Data Analysis Software and Systems I, ed. D. M. Worrall, C. Biemesderfer, \& J. Barnes (San Francisco, CA: ASP), 245

Lin, D. N. C., Papaloizou, J. C. B., Terquem, C., Bryden, G., \& Ida, S. 2000, in Protostars and Planets IV, ed. V. Mannings, A. P. Boss, \& S. S. Russell (Tucson, AZ: Univ. Arizona Press), 1111

Liseau, R., et al. 2008, A\&A, 480, L47

Lissauer, J. J. 2005, Space Sci. Rev., 116, 11

Lisse, C. M., Beichman, C. A., Bryden, G., \& Wyatt, M. C. 2007, ApJ, 658, 584

Morel, M., \& Magnenat, P. 1978, A\&AS, 34, 477

Moro-Martín, A., \& Malhotra, R. 2005, ApJ, 633, 1150

Moro-Martín, A., et al. 2007, ApJ, 658, 1312

Raymond, S. N., Barnes, R., Armitage, P. J., \& Gorelick, N. 2008, ApJ, 687, L107

Santos, N. C., Israelian, G., \& Mayor, M. 2001, A\&A, 373, 1019

Skrutskie, M. F., et al. 2006, AJ, 131, 1163

Su, K. Y. L., et al. 2006, ApJ, 653, 675

Trilling, D. E., et al. 2008, ApJ, 674, 1086

Udry, S., \& Santos, N. C. 2007, ARA\&A, 45, 397

Valenti, J. A., \& Fischer, D. A. 2005, ApJS, 159, 141

Wright, J. T., Marcy, G. W., Butler, R. P., \& Vogt, S. S. 2004, ApJS, 152, 261

Wyatt, M. C. 2008, ARA\&A, 46, 339

Wyatt, M. C., Clarke, C. J., \& Greaves, J. S. 2007, MNRAS, 380, 1737 